\title{
Prevention and Control Strategies for Parasitic Infections in the Korea Centers for Disease Control and Prevention
}

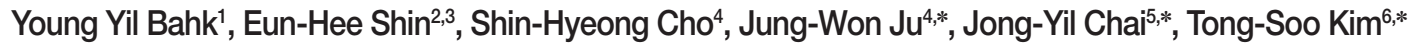 \\ ${ }^{1}$ Department of Biotechnology, College of Biomedical and Health Science, Konkuk University, Chungju 27478, Korea; ${ }^{2}$ Department of Parasitology \\ and Tropical Medicine, Seoul National University College of Medicine, and Institute of Endemic Diseases, Seoul National University Medical Research \\ Center, Seoul 03080, Korea; ${ }^{3}$ Seoul National University Bundang Hospital, Seongnam 13620, Korea; ${ }^{4}$ Division of Vectors and Parasitic Diseases, \\ Korea Centers for Disease Control and Prevention, Osong 28159, Korea; ${ }^{5}$ Korea Association of Health Promotion, Seoul 07653, Korea; \\ ${ }^{6}$ Department of Parasitology and Tropical Medicine, Inha University School of Medicine, Incheon 22212, Korea
}

\begin{abstract}
Korea is successfully controlled intestinal parasitic infections owing to economic development and high health consciousness. The Division of Vectors and Parasitic Diseases (formerly the Division of Malaria and Parasitology) is in the Center for Laboratory Control of Infectious Diseases of the Korea Centers for Disease Control and Prevention. It has been the governmental agency responsible for controlling and leading scientific research on parasitic diseases. The Division of Vectors and Parasitic Diseases has conducted and funded basic research and disseminated the research results to various medical fields, ultimately promoting public health in Korea. Among the noteworthy achievements of this division are the national surveillance of healthcare-associated parasitic infections, prevention and control for parasitic infections, and the elimination of lymphatic filariasis from Korea. On a broader scale, the division's research programs and academic supports were influential in preventing and treating infectious parasitic diseases through public policies and laws. In this review, we summarize the past and present role of the Division of Vectors and Parasitic Diseases in preventing and treating infectious parasitic diseases in Korea.
\end{abstract}

Key words: Surveillance of parasite, parasite control, KCDC

\section{INTRODUCTION}

Life forms on earth are always exposed to parasites and thus are constantly being reinfected in a chain reaction mode. Parasitic diseases inflict considerable socio-economic losses to society. Zoonotic parasites can result in disease manifestations in humans and substantial economic losses to livestock populations. According to the World Health Organization (WHO), over 3 billion people around the world suffer from one or more parasitic diseases, which are widespread and a leading cause of morbidity and mortality in populations [1]. Parasites have infected humans for thousands of years, and nearly all known human-specific parasites have been found in ancient feces [2,3]. Indeed, no organism is protected against parasites. Anti-parasitic drugs are effective in minimizing parasitic infec-

\footnotetext{
- Received 15 August 2018, revised 15 October 2018, accepted 16 October 2018. *Corresponding authors (tongsookim@inha.ac.kr; cjy@snu.ac.kr; jupapa@korea.kr) (C) 2018, Korean Society for Parasitology and Tropical Medicine This is an Open Access article distributed under the terms of the Creative Commons Attribution Non-Commercial License (http://creativecommons.org/licenses/by-nc/4.0) which permits unrestricted non-commercial use, distribution, and reproduction in any medium, provided the original work is properly cited.
}

tions. However, this is not a long-term solution. Ameliorating the effects of these diseases may include attempts of eradicating specific diseases at a global level, eliminating them at a national or local level, or controlling them to minimize infections. Generally, it is impossible to adequately protect the health of the public without addressing infectious disease problems. Traditionally, public health burdens include infectious diseases such as parasitic diseases, and a public health system would be required to use a demographic approach. A recent literature survey of human pathogens found more than 1,400 different species, of which more than half are known to be zoonotic, i.e., able to infect other host species, thus demonstrating the need for one health approach to many human and animal diseases [4]. Thus far, beyond communicative diseases, neglected tropical diseases (NTDs) including some parasitic infections are emerging or resurging as a result of changes in public health policy, drug resistance, social pattern changes, and genetic mutations in pathogens; NTDs are a group of chronic disabling infections affecting more than 1 billion people worldwide [5]. Therefore, the control of NTDs and traditional infectious diseases has been identified as a major bur- 
den worldwide [6]. In addition, global warming has caused climate changes, disrupting the biodiversity of various species that depend on a proper ecosystem. Global warming can alter the geographic distribution and intensity of the transmission of vector-borne diseases. The transmitted parasites usually benefit from increased temperatures as both their reproduction and development are accelerated.

In 1966, the Korean National Assembly passed the "Parasite Diseases Prevention Act". With the passage of this law, the Korea National Institute of Health (KNIH) became the leading governmental agency to address problems associated with parasitic infections. Generally, it is impossible to adequately protect the health of any nation without addressing infectious disease problems that occur elsewhere in the world. The KNIH has long engaged in efforts to prevent and control parasitic diseases and provides training, technical assistance, and other support to increase the capacity of country-based researchers to optimize their effectiveness. Population health improvement often involves policy changes that result from complex efforts. Systems, policies, and procedures to measure and prevent parasite-associated infections are essential for a comprehensive response to eliminate parasitic diseases.

The initial organization for the management of infectious diseases in Korea was Korea Institute of Prevention Disease, which was established in September 1945. The management of roundworms, flukes, etc., which were prevalent in Korea, started in 1959. Since then, there have been several organizational changes. The Division of Malaria and Parasitology (DMP) was an organization of the KNIH until 2017. The DMP was established in 1963 in the Department of Hygiene, and it was an organization part of the Department of Virology from the 1970s onwards. In 1997, the DMP was merged with the Division of Insect according to the re-organization of the KNIH. In 2003, following the establishment of the Korea Centers for Disease Control and Prevention (KCDC), the DMP was again separated from the Division of Insect. Following the re-organization of the KCDC in 2017, the DMP has been integrated with the Division of Insect and renamed as the Division of Vectors and Parasitic Diseases (DVPD). Currently, the DVPD belongs to the Center for Laboratory Control of Infectious Diseases (CLCID) of the KCDC.

In this review, we briefly describe the role of the DVPD (formerly known as the DMP) in the CLCID of the KCDC. In addition, we examine its cooperative efforts with foreign organizations involved in parasite management projects for disease control in countries including the USA, Japan, UK, Switzerland, and
EU nations from 2006 to 2015. We obtained basic information on the mid-term and long-term plans of the DVPD of the KCDC (DMP, KNIH). Due to improvements in epidemiological surveillance over the last 4 decades and the systematic management of populations in highly endemic areas, the prevalence of parasitic infections nationwide is currently very low. Recent cases of parasitic diseases are mainly attributed to the transmission of fish/food-borne trematodes. This review systematically examines the role of the Center for Infectious Diseases Research in public health-associated parasite management and control.

\section{SURVEILLANCE OF PARASITE-ASSOCIATED INFECTIONS IN KOREA}

Intestinal parasitic infections by helminth and protozoa are among the most prevalent infections worldwide [7]. In Korea, intestinal helminth infections were highly prevalent in the past, which were one of the major public health burdens [8]. During the 1950s and 1960s, Korea was one of the poorest countries in the world. Based on the "law for Prevention of Parasitic Diseases" (Parasite Diseases Prevention Act) by the Korean Government, a national control program was implemented by targeting the school-age population in the country from 1969 to 1994. Infection prevention and control measures and practices can reduce the spread of parasites among members of the public. Surveillance programs should promote the implementation of evidencebased infection prevention and control practices to reduce the incidence of these diseases, decrease transmission, and ultimately improve public health. The surveillance of healthcare-associated parasitic infections and infection prevention and control activities would not only help manage parasitic diseases but also contribute to the overall economic development. Therefore, accurate surveillance is important to control parasitic diseases. In the past, the lack of adequate systems and infrastructure for accurate assessment and infection prevention and control in Korea contributed the spread of parasitic diseases, and Korea was one of the countries with a high intestinal helminth infection rate, resulting in a high public health risk $[9,10]$. Accurate estimates of the burden of parasite-associated infections can be obtained based on reliable and routine standardized surveillance data. Nationwide surveys on the prevalence of infections revealed that the egg-positive rate of helminth infections was $90.5 \%$ in $1969,84.3 \%$ in 1971 , $63.2 \%$ in $1976,41.1 \%$ in $1981,12.9 \%$ in $1986,3.8 \%$ in 1992 , $2.4 \%$ in $1997,3.7 \%$ in 2004, and $2.6 \%$ in 2012 [11,12]. Nevertheless, Korea is an Asian country that has successfully controlled 
intestinal parasitic infections owing to economic development, as shown by the results of national surveys every 5-7 years since 1971. The data in 1971 revealed an overall helminth egg-positive rate of $84.3 \%$ and an accumulated egg-positive rate of $147.1 \%$, indicating that Koreans had at least one or more types of intestinal parasitic infections. According to the 8th Prevalence of Intestinal Parasitic Infections in Korea (a nationally approved statistical report), despite a decrease in intestinal parasitic infections, the egg-positive rate was still 2.6\% in 2012, fish/food-mediated parasitic infections continued to occur, and infection by Trichuris trichiura (a soil-transmitted parasite) was gradually increasing [12]. The Ministry of Health and Welfare (MOHW) in Korea, with support from the Center for Infectious Diseases Research of the $\mathrm{KNIH}$, has conducted parasitic infection surveillance with the objective of strengthening infection prevention and control practices and characterizing parasitic infections by helminths and protozoa. Accordingly, all districts promptly and routinely report key parasite indicators to support passive and active surveillance and monitoring and evaluation systems. The surveys identified the status of parasitic infections at the national level and collected a large amount of data that could be used to determine the direction and development of the parasite management system.

Cases of infections due to global warming or seasonal changes have been reported, and malaria cases have emerged and are increasing over the past 20 years $[13,14]$. The continued and systematic strengthening of control activities against vivax malaria in Korea has been conducted considering that the number of vivax malaria cases has reached a steady-state level after its re-emergence in 1993 [14]. Furthermore, infections from fish/ food-borne parasites continue to occur, threatening human health. A national strategy to eliminate parasites has been devised to ensure that the knowledge, attitudes, and practices of the population regarding parasitic diseases are improved through education, communication, etc. An in-depth assessment of the impact of parasites on human health as well as the design of optimal protocols for parasite control would greatly depend on the robustness of the data obtained. In Korea, the burden of health-associated parasitic infections can be determined based on reliable and routine standardized surveillance data in the form of a nationally approved statistical report.

\section{PARASITIC INFECTION PREVENTION AND CONTROL BY THE CLCID OF THE KCDC}

Until the 1970s, most Koreans had high levels of intestinal parasitic infections, mostly from soil-transmitted helminths such as Ascaris lumbricoides, Trichuris trichiura, hookworms (Ancylostoma duodenale and Necator americanus), and Trichostrongylus orientalis $[15,16]$. In particular, A. lumbricoides (a representative intestinal parasite and an index of soil-transmitted helminths) demonstrated an egg-positive rate of 54.9\% in 1971. South Korea was an agricultural society that used human manure as fertilizer and had a poor sanitary and living standard with a variety of issues that can cause intestinal parasitic infections. At the 1st Foodborne Diseases Burden Epidemiology Reference Group (FERG) meeting in 2006 by WHO, the Parasitic Diseases Task Force identified parasites that could be transmitted via food to humans. From this list, parasites that could cause a substantial burden of disease were identified and prioritized. These included intestinal protozoa (Giardia, Entamoeba, and Cryptosporidium spp.), Fasciola spp., Trichinella spp., Toxoplasma gondii, Echinococcus spp., Opisthorchis spp., Clonorchis spp., Taenia solium, Anisakis simplex, and Ascaris lumbricoides [17]. Estimating the global disease burden caused by parasites and other hazards is challenging because of complex transmission pathways and the various health outcomes. In terms of parasitic disease management, the DMP mainly focused on epidemiological surveys and the treatment of common intestinal parasites in Korea prior to 2000. Subsequently, the investigation on parasitic infection and treatment was continued with additional research and development by introducing specimen-monitoring systems for indigenous parasitic diseases, re-exporting diseases, and inflow diseases. The DVPD has participated actively in these projects. The parasitic infection prevention and control efforts of the KCDC were successful by 2008. Lymphatic filariasis caused by infection with Brugia malayi, which results in elephantiasis, was first recorded in Korea in 1927 [18]; it was reported in the Chungcheongnamdo Province and Jeju Special Self-Governing Province [19]. Until the early 2000s, lymphatic filariasis was prevalent in the coastal areas in Korea [20]. Through sustained efforts with investigation and treatment, filariasis was officially eliminated in 2008 [21-24]. With a constant national parasitic infection incidence of less than 1 case per 1,000 population at risk (except fish/food-borne parasites, especially C. sinensis and heterophyids) in Korea, domestic parasitic infection rates have decreased to the level of advanced countries. As the WHO has reported that infection with C. sinensis leads to an increased risk of cholangiocarcinoma [25], which is a malignant tumor of biliary tract epithelia, a management system has been established to 
acquire clinical evidence related to cholangiocarcinoma through the development of a highly sensitive diagnostic tools (e.g. CT, MRI, nanoparticle-based detection etc.). The continued and systematic strengthening of control activities against vivax malaria in South Korea has been conducted considering that the number of vivax malaria cases has reached a steady-state level (less than 1.4 incidence rate [per 100,000]) after its re-emergence in 1993 [14].

The need for standardized parasitic infection prevention and treatment has reduced, and screening and diagnosis are difficult due to low prevalence. However, climate changes due to global warming could likely destroy or modify the natural habitat of parasites. Therefore, long-term planning is needed to overcome parasitic threats. The implementation of systematic healthcare-associated infection surveillance and infection prevention and control programs in combination with efforts to accurately report parasitic diseases will improve the detection and prevention of parasitic diseases in Korea and other countries in the region. In addition, internet-based education, defined as using to prevent and access learning materials, helps to interact with others and enhance the learning process [26]. The KCDC has provided internet-based education since 2010; an internet-based platform is used to deliver free learning materials about parasites (http://www.nih-cyber.or.kr).

\section{STATUS OF THE ACADEMIC SUPPORT SERVICES AND RESEARCH PROGRAMS OF THE CLCID}

As part of the national response to parasitic diseases, the DVPD was established in the CLCID of the KCDC for the systematic assessment and improvement of infection prevention and control practices and for the standardized surveillance of healthcare-associated parasitic infections in 2003. One of its objectives was to develop models that can serve as the foundation of a sustainable Korean national network for the standardized implementation and strengthening of infection prevention and control practices and for the standardized reporting of healthcare-associated parasitic infections. Collaborations with scientists who are experts in the relevant fields are known to provide information that can clarify the pathophysiology of diseases. These support services can strengthen the academic institute, contribute to new diagnostic strategies, and, perhaps most importantly, offer hope to patients and their family.

The academic support services provided from 2006 to 2015 involved a total of 102 subjects including protozoa (8 species), nematodes ( 5 species), trematodes ( 3 species), cestodes (1 species), etc. (Fig. 1). Based on the data on parasitic infections in Korea, the proportion for studies on malaria and C. sinensis was $58.8 \%$. The studies included immunopathology (24\%),

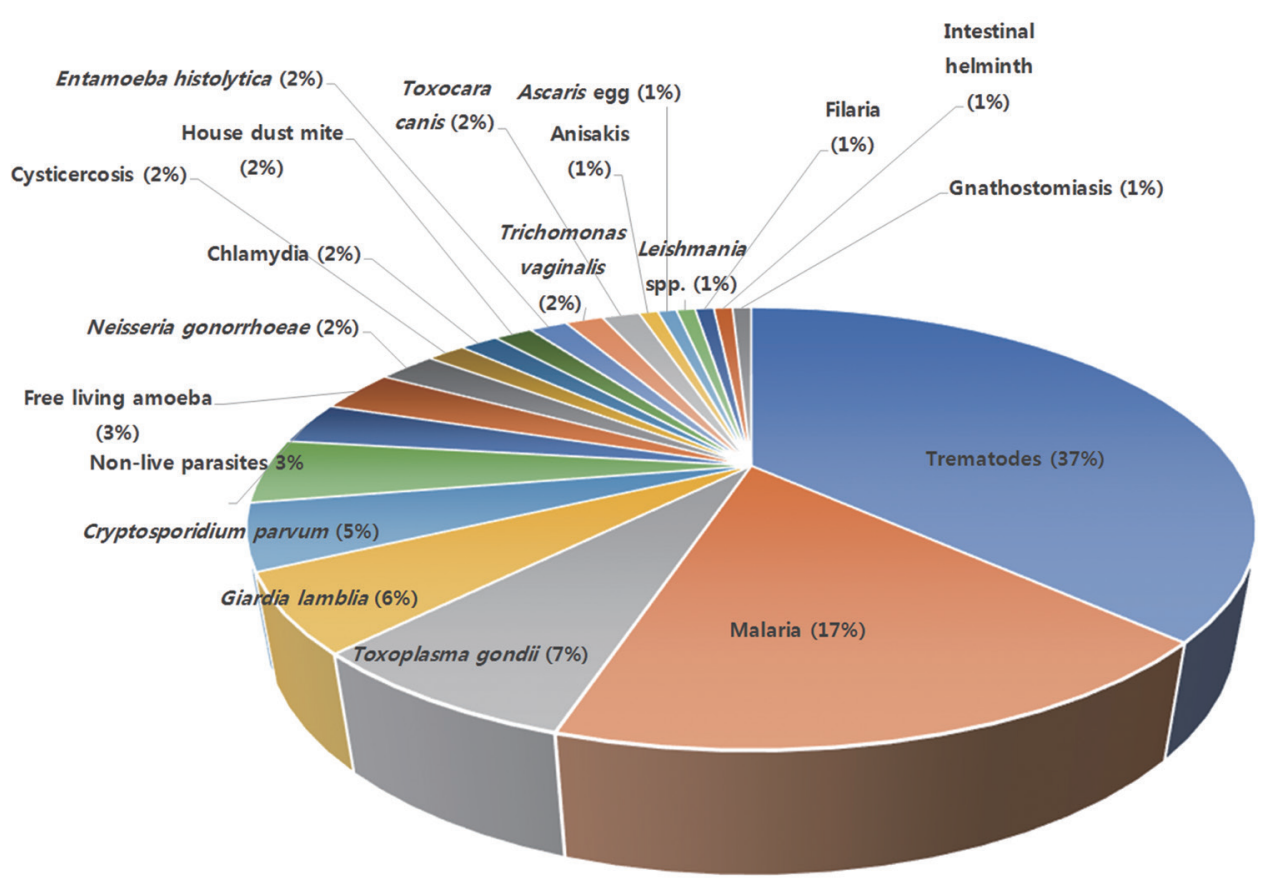

Fig. 1. Distribution of academic research supports provided by KCDC. 
ecological studies (18\%), molecular biological and genetic studies (15\%), policy studies (11\%), and other diagnostic studies $(25 \%)$. As the diseases caused by these parasites are persistent in Korea, studies to monitor and investigate the nature of these parasites are necessary for the establishment of healthcare-associated policies. Overall, 39\% of the studies were focused on basic scientific research, such as characterizing the interrelationships between the host and parasites and the effect on the host system. On the other hand, $48 \%$ of the studies were involved in improving parasitological diagnostic techniques and trend analysis in Korea.

In addition, $11 \%$ of investment was on policy development, which encouraged studies for the purpose of policy establishment. The incidence of imported and unproven cases of parasitosis has markedly increased in recent years because of improvements in the performance and availability of specific diagnostic techniques, the immigration of infected persons, and increased intercontinental travel.

Intramural research of the DVPD from 2011 to 2017 were a total 21 projects involving vivax malaria, mosquitoes, therapeutic agents diagnostic for parasitic infection, C. sinensis, toxoplasmosis, dengue fever, tsutsugamushi disease, and helminths. The main priority is to provide epidemiological, diagnostic, and clinical assistance to healthcare providers and researchers and increase the awareness towards parasitic diseases in Korea. Another critical objective is to support the elimination of malaria and targeted NTDs by obtaining critical strategic information (surveillance, monitoring, and evaluation, and research) to strengthen the scientific evidence. The KCDC research program is needed to address the needs of individuals and families with rare or multisystem diseases who remained underdiagnosed after an extensive evaluation. The research on parasitic diseases can provide value for patients and their family and the institution and contribute to the advancement of medical science.

\section{INTERNATIONAL OUTBREAK ASSISTANCE AND COOPERATION WITH FOREIGN INSTITUTES}

International outbreak assistance is an integral function and one of the priorities of the Center for Infectious Diseases Research. Outbreaks offer opportunities to learn more about the dynamics of disease transmission, the effectiveness of prevention and treatment strategies, and the risk factors for severe or fatal diseases. Generally, the knowledge gained in an investigation can be applied to various areas. This center is cooperating with several international organizations, including those in the USA, Japan, China, Myanmar, and Thailand, UK, Switzerland, and EU. It has obtained valuable data for mid-term to long-term plans for managing the infectious parasitic diseases by referring their achievements. Korea achieved the parasitic disease prevention and control; thus, it plays an important role in the fight against diseases in the world.

\section{SENTINEL SURVEILLANCE OF IMPORTED PARASITIC DISEASES AND SYSTEMATIC CONTROL ACTIVITIES IN TERMS OF TRAVEL}

Imported parasitic diseases include tropical diseases, which are infectious diseases that thrive in hot and humid conditions. Owing to rapid economic development and the mass movement of people, the risk of infectious parasitic diseases that were not endemic in Korea has greatly increased. Cases of imported parasitic diseases have revealed the weaknesses in controls against imported parasitic diseases in Korea: (1) travelers are not aware of the prevention and protection methods against infectious parasitic diseases in endemic regions; (2) due to the rarity of these imported diseases, medical organizations lack diagnostic capacity, which usually causes a significant delay in diagnosis; (3) the unavailability of specific medicines in Korea could complicate treatment initiation. To help protect Koreans against unwanted tropical parasitic diseases, the DVPD in the CLCID of the KCDC monitors the importation of parasites into Korea through the sentinel surveillance of diseases including leishmaniasis (L. tropica, L. major, L. donovani, L. infantum, etc.), babesiosis (B. microti, B. bigemina, etc.), African trypanosomiasis (T. gambiense, T. rhodesiense, etc.), schistosomiasis (S. japonicum, S. mansoni, S. haematobi$u m$, etc.), Chagas disease (Trypanosoma cruzi), angiostrongyliasis (Angiostrongylus cantonensis), gnathostomiasis (Gnathostoma spinigerum, etc.), filariasis (Wuchereria bancrofti, Brugia malayi, Onchocerca volvulus, Loa loa, Dirofilaria immitis, Dirofilaria repens), hydatidosis (Echinococcus granulosus and E. multilocularis), toxoplasmosis (Toxoplasma gondii), and dracunculiasis (Dracunculus medinensis) [27].

To prevent unwanted imported parasitic diseases, specific measures have been implemented by the KCDC: (1) a database of imported parasitic diseases including those already imported and those that would potentially be imported in the future has been established (for example: http://pathod.cdc. go.kr for liver fluke and http://ToxoDB.org for toxoplasmosis); 
(2) medical organizations and quarantine stations have established a mechanism for providing medical counseling services; (3) the diagnostic capacity for imported parasitic diseases has been strengthened, which includes instruction in medical schools and the training of special doctors in hospitals; (4) a mechanism to provide specific drugs rapidly for prompt treatment has been established.

Re-emerging Parasitic diseases, which were eradicated from the public previously, remain a major cause of sickness worldwide. Changes in epidemiological factors such as travel habits, technology, and climate, combined with the decreased effectiveness of some measures of disease control, have altered the etiology of parasitosis. The patterns of imported parasitic diseases are affected by several factors, including increased international travel, risky travel patterns, changes in the purpose of travel, increased parasitic disease transmission in certain areas, and the increased drug resistance of the parasite. Travelers will encounter various infectious agents at their destination that would differ largely from those at home. Indeed, travel plays a critical role in the globalization of infection. Imported parasitic diseases threaten not only non-immune travelers but also settled immigrants in Korea and their children, who are born in a non-endemic country. In addition, imported parasitosis may be attributed to insects such as mosquitoes or the eating/ drinking patterns of those who travel abroad. In the past, parasitic infections were largely associated with employment in the East Asia and Middle East, which increased the cases of malaria. The most common parasitic infection caused by traveling abroad is malaria, followed by babesiosis, gnathostomiasis, hydatidosis, and leishmaniasis [27]. Since 2001, dengue fever transmitted by Aedes albopictus mosquitoes has emerged as an infectious disease in Korea. Between 2006 and 2010, 367 suspected cases of dengue fever were reported [28]. The 1st case in Korea was reported in 1995, involving a woman who traveled to Sri Lanka [29]. Recently Aedes spp. are also found in Korea and dengue fever can be potentially transmitted in Korea as autochthonous introductory case. In general, specific vaccines have not been developed for most parasitic diseases, thus resulting in chronic outcomes. As there is the possibility of developing serious and fatal symptoms, it is necessary to establish an education and monitoring system and to develop post-travel reporting channels to prevent tourists from being infected with the diseases (Case Definitions for National Notifiable Infectious Diseases). Currently, the Center for Infectious Diseases Research, KCDC is responsible for the national con- trol of parasitic diseases, which are classified as group III diseases that require surveillance. As the number of tourists traveling to developing countries increases, additional systematic diagnostic referral systems are required. The center has a system for monitoring parasitic infections in response to the increasing demand for international travel. In Korea, parasitic infection cases in hospitals or clinics, especially the number of patients, need to be reported to the Local Community Health Center (CHC), as required by law. CHCs have to report these cases periodically to the Division of Infectious Disease Surveillance (DIDS) at the KCDC [30]. Accordingly, it provides the public with detailed information about parasitic infections in other countries. Imported infectious diseases can not only affect the health of individuals but also cause the spread of an endemic disease in another country. Therefore, 13 quarantine stations have been established to carry out quarantine checks of ships, aircraft, and passengers nationwide to prevent the spread of these diseases.

\section{LOOKING FORWARD AND FUTURE DIRECTIONS}

The DVPD in the CLCID of the KCDC, the nation's leading agency on parasitic diseases, has conducted research on a wide range of topics, including the genetics, diagnosis, epidemiology, health risks and benefits, prevention, and treatment of parasites. Considering the technological advances in high-throughput screening and data collection, research on the biodiversity of parasites should be performed to develop effective management measures against infectious parasitic diseases. Instead of focusing on basic scientific research, more efforts should be dedicated to the application of existing tools and knowledge to improve the current health system. In addition, analyses of the correlation between parasitic infections and chronic diseases and of parasitic substances for the potential therapeutic treatment of chronic diseases should be performed. Accordingly, joint projects with statisticians should be conducted to create predictive scenarios and explore big data. Additionally, it is necessary to establish a regional research center for the DMP to investigate the rate of parasitic infections and identify the dynamic patterns of parasites with respect to the time zone, region, and environment. A global database for international healthcare cooperation should be developed for sharing important information with researchers. Nevertheless, it can also be used to address difficulties in diagnosis due to low prevalence as well as infections caused by changes in the cli- 
mate due to global warming.

To achieve success in these and other endeavors, the DVPD in the CLCID of the KCDC should continue to cooperate with individuals and agencies within the KCDC and the relevant communities in Korea and around the world. The prevention and treatment of parasitic infections would help to decrease health disparities.

\section{ACKNOWLEDGMENT}

This study was supported by a research grant (2016-E54001-00) from the Korea Centers for Disease Control and Prevention in the Republic of Korea (TSK).

\section{CONFLICT OF INTEREST}

The authors declare that they have no competing interests.

\section{REFERENCES}

1. World Health Organization. Sustaining the Drive to Overcome the Global Impact of Neglected Diseases: Second WHO Report on Neglected Diseases. Geneva, Switwerland. World Health Organization. 2013.

2. Araujo A, Reinhard K, Ferreira LF, Pucu E, Chieffi PP. Paleoparasitology: the origin of human parasites. Arquivos de neuropsiquiatria 2013; 71: 722-726 (in Portuguese).

3. Shin DH, Chai JY, Hong JH, Seo M. Historical details about the meat consumption and Taeniases in Joseon period of Korea. Korean J Parasitol 2017; 55: 457-460.

4. Woolhouse ME, Gowtage-Sequeria S. Host range and emerging and reemerging pathogens. Emerg Infect Dis 2005; 11: 1842-1847.

5. The Lancet. Neglected tropical diseases: becoming less neglected. Lancet 2014; 383: 1269.

6. Molyneux DH. Combating the "other diseases" of MDG 6: changing the paradigm to achieve equity and poverty reduction? Trans R Soc Trop Med Hyg 2008; 102: 509-519.

7. Savioli L, Albonico M, Engels D, Montresor A. Progress in the prevention and control of schistosomiasis and soil-transmitted helminthiasis. Parasitol Int 2004; 53: 103-113.

8. Hong ST, Chai JY, Choi MH, Huh S, Rim HJ, Lee SH. A successful experience of soil-transmitted helminth control in the Republic of Korea. Korean J Parasitol 2006; 44: 177-185.

9. Seo BS, Lee SH, Cho SY, Chai JY, Hong ST, Han IS, Sohn JS, Cho BH, Ahn SR, Lee SK, Chung SC, Kang KS, Shim HS, Hwang IS. An epidemiologic study on clonorchiasis and metagonimiasis in riverside areas in Korea. Korean J Parasitol 1981; 19: 137-150.

10. Seo BS. Epidemiology and control of Ascariasis in Korea. Korean J Parasitol 1990; 28 (suppl): 49-61.
11. Ministry of Health and Social Affairs and Korean Association of Parasite Eradication. Prevalence of intestinal parasitic infections in Korea - The 2nd report. Seoul, Korea. 1976, pp 1-147 (In Korean).

12. Korea Centers for Disease Control and Prevention. Korea Association of Health Promotion: Prevalence of intestinal parasites in Korea - The 8th Report. Seoul, Korea. 2012.

13. Traill LW, Lim ML, Sodhi NS, Bradshaw CJ. Mechanisms driving change: altered species interactions and ecosystem function through global warming. J Anim Ecol 2010; 79: 937-947.

14. Bahk YY, Lee HW, Na BK, Kim J, Jin K, Hong YS, Kim TS. Epidemiological characteristics of re-emerging vivax malaria in South Korea. Korean J Parasitol Revised for publication.

15. Seo BS, Rim HJ, Loh IK, Lee SH, Cho SY, Park SC, Bae JW, Kim JH, Lee JS, Koo BY, Kim KS. Study on the status of helminthic infections in Koreans. Korean J Parasitol 1969; 7: 53-70 (in Korean).

16. Kim CH, Park CH, Kim HJ, Chun HB, Min HK, Koh TY, Soh CT. Prevalence of intestinal parasites in Korea. Korean J Parasitol 1971; 9: 25-38 (in Korean).

17. 17 World Health Organization. First formal meeting of the Foodborne Disease Burden Epidemiology Reference Group (FERG): implementing strategy, setting priorities and assigning the tasks. Geneva, Switzerland. World Health Organization. 2007, pp 1-43.

18. Yun IS. Elephantiasis due to filarial in Korea. Chosen Iggakai Zasshi 1927; 76: 326-334.

19. Oh HY. Filariasis in Korea. Chinese Med J 1929; 43: 16-21.

20. Chai JY Lee SH, Choi SY, Lee JS, Yong TS, Park KJ, Yang KA, Lee KH, Park MJ, Park HR, Kim MJ, Rim HJ. A survey of Brugia malayi infection on the Heuksan islands, Korea. Korean J Parasitol 2003; 41: 69-73.

21. Korea National Institute of Health, Korea Centers for Disease Control and Prevention. National documentation for certification: elimination of lymphatic filrariasis in Korea. Seoul, Korea. Korea National Institute of Health, Korea Centers for Disease Control and Prevention. 2007.

22. Cheun HI, Lee JS, Cho SH, Kong Y, Kim TS. Elimination of lymphatic filariasis in the Republic of Korea: an epidemiological survey of formerly endemic areas, 2002-2006. Trop Med Int Health 2009; 14: 445-449.

23. Cheun H-I, Kong Y, Cho SH, Lee JS, Chai JY, Lee JS, Lee JK, Kim TS. Successful control of lymphatic filariasis in the Republic of Korea. Korean J Parasitol 2009; 47: 323-335.

24. Cho SH, Ma DW, Koo BR, Shin HE, Lee WK, Jeong BS, Chu C, Lee WJ, Cheun HI. Surveillance and vector control of lymphatic filariasis in the Republic of Korea. Osong Public Health Res Perspect 2012; 3: 145-150.

25. Kim TS, Pak JH, Kim JB, Bahk YY. Clonorchis sinensis, an oriental liver fluke, as a human biological agent of cholangiocarcinoma: a brief review. BMB Rep 2016; 49: 590-597.

26. Wutoh R, Boren SA, Balas EA. eLeaning: a review of internetbased continuing medical education. J Contin Educ Health Prof 
2004; 24: 20-30.

27. Korean Centers for Diseases Control and Prevention. Infectious Diseases Surveillance System [Internet]; Available from: http:// is.cdc.go.kr.

28. Park JH, Lee DW. Dengue fever in South Korea, 2006-2010. Emerg Infect Dis 2012; 18: 1525-1527.
29. Lee CJ, Kim HR, Kim MJ. A case of imported dengue hemorrhagic fever. Korean J Infect Dis 1995; 27; 404-406 (in Korean).

30. Korean Centers for Diseases Control and Prevention. Guidelines for Malaria Prevention and Control 2016. 2016, pp 1-177 (in Korean). 